\title{
NMR and molecular modelling studies on elastase inhibitor-peptides for wound management
}

\author{
Sandra Cerqueira Barros ${ }^{a}$, Ricardo O. Louro ${ }^{b}$, Nuno M. Micaêlo ${ }^{a}$, José Alberto Martins ${ }^{a}$, \\ João Carlos Marcos ${ }^{\mathrm{a}}$, Artur Cavaco-Paulo ${ }^{\mathrm{c}, *}$ \\ ${ }^{a}$ Centro de Química, Universidade do Minho, Campus de Gualtar, 4710-057 Braga, Portugal \\ ${ }^{\mathrm{b}}$ Instituto de Tecnologia Química e Biológica (ITQB), Universidade Nova de Lisboa, Av. da Republica (EAN), 2780-157 Oeiras, Portugal \\ ${ }^{\mathrm{c}}$ Instituto de Biotecnologia e Bioengenharia (IBB), Centro de Engenharia Biológica, Universidade do Minho, Campus de Gualtar, 4710-057 Braga, Portugal
}

\section{A R T I C L E I N F O}

\section{Article history:}

Available online 4 March 2013

\section{Keywords:}

Elastase inhibitor-peptides

SLPI

Phosphorylation

2D-NMR

Molecular docking

\begin{abstract}
A B S T R A C T
Proteases play an important and critical role in the physiological process of wound repair. However, excessive and unregulated release of proteolytic enzymes (e.g., elastase) mediates abnormal degradation of healthy tissues, which leads to inflammatory disorders such as chronic wounds. Thus, it is of therapeutic interest to develop novel synthetic inhibitor-peptides of elastase, which can restore the balance between the free enzyme and the endogenous inhibitors in chronic wounds. In previous works, we have reported two different drug delivery systems to release novel elastase inhibitors to the wound site. In both systems synthetic peptides (KRCCPDTCGIKCL-Pep4 and KRMMPDTMGIKML-Pep4M) based on the primary structure of the endogenous elastase inhibitor, secretory leucocyte protease inhibitor, were used as active material. Phosphorylation of the reported peptides prompts significant structural differences, which reflects in distinct inhibitory capacity towards elastase. These structural modifications were prompted by electrostatic interactions and hydrogen bonds established from the peptide phosphoresidue. The current study was also extended to another synthetic peptide (WCTASVPPQCY-PepBBI) that is based on the reactive loop of another elastase inhibitor, the Bowmen-Birk inhibitor. PepBBI, phosphorylated and non-phosphorylated, displays similar behaviour to Pep4 and Pep4M. The structural modifications reported herein were evaluated by two-dimensional nuclear magnetic resonance and molecular modelling approaches.
\end{abstract}

() 2013 Elsevier Ltd. All rights reserved.

\section{Introduction}

Normal wound healing is a controlled balance between repair processes that lead to new tissue formation and destructive processes, which are essential to remove damaged tissue $[1,2]$. The perfect control of the biological processes involved in healing leads to normal wound repair. However, any alteration in these physiological processes may lead to a non-healing state and the formation of a chronic wound [1]. Chronic wounds fail to progress through the normal stages of healing and therefore enter a state of pathologic inflammation [2,3]. The prolonged inflammatory phase

Abbreviations: MMPs, matrix metalloproteinases; PPE, porcine pancreatic elastase; SLPI, secretory leukocyte protease inhibitor; Pep4, KRCCPDTCGIKCL; Pep4(P), Pep4 phosphorylated; Pep4M, KRMMPDTMGIKML; Pep4M(P), Pep4M phosphorylated; PepBBI (Bowman-Birk inhibitor), WCTASVPPQCY; PepBBI(P), PepBBI phosphorylated; Thr(P), Phosphothreonine; 2D NMR, two-dimensional nuclear magnetic resonance; COSY, 2D correlated spectroscopy; TOCSY, 2D total correlated spectroscopy; NOESY, nuclear Overhauser enhancement and exchange spectroscopy; NOE, nuclear Overhauser enhancement or nuclear Overhauser effect; RMSD, root mean square deviation.

* Corresponding author.

E-mail address: artur@deb.uminho.pt (A. Cavaco-Paulo). characteristic of chronic wounds results in exuberant response of neutrophils [4]. Neutrophils invade the inflammatory sites and their massive accumulation at the inflammation site mediates the release of high amounts of proteases (concentrations reaching millimolar range) and free radical generating enzymes that become deleterious to the healing process [4-7]. The proteolytic environment of the wound resulting from high neutrophil concentrations is a combination of cationic serine proteases (e.g., human neutrophil elastase (HNE)) and matrix metalloproteinases (e.g. collagenase MMP-8 and gelatinase MMP-9) [4,6]. These two families of proteases, when at normal levels, augment wound healing. However, at high levels are associated with the degradation of important growth factors and major proteins of the extracellular matrix necessary for wound healing. Among the proteases released from neutrophils, human neutrophil elastase (HNE) is capable of cleaving tissue proteins such as elastin, which plays a major function in the arteries, skin and ligaments, collagen (Types I-V, IX and $\mathrm{XI}$ ), fibrin, fibronectin, cartilage, proteoglycans, and cytokines $[5,8,9]$. Elastase action is modulated by multiple endogenous inhibitors, such as $\alpha 1$-protease inhibitor, secretory leucocyte protease inhibitor (SLPI) and elafin. Still, under pathological conditions the oxidative inactivation of these natural protease inhibitors takes 


\section{SLPI primary structure}

\begin{tabular}{|llllllllllllllllllll}
\hline S & G & K & S & F & K & A & G & V & C $^{10}$ & P & P & K & K & S & A & Q & C & L & R $^{20}$ \\
Y & K & K & P & E & C & Q & S & D & W $^{30}$ & Q & C & P & G & K & K & R & C & C & P $^{40}$ \\
D & T & C & G & I & K & C & L & D & $\mathrm{P}^{50}$ & V & D & T & P & N & P & T & R & R & $\mathrm{K}^{60}$ \\
P & G & K & C & P & V & T & Y & G & Q $^{70}$ & C & L & M & L & N & P & P & N & F & $C^{80}$ \\
E & M & D & G & Q & C & K & R & D & L $^{90}$ & K & C & C & M & G & M & C & G & K & S $^{100}$ \\
C & V & S & P & V & K & $A^{107}$ & & & & & & & & & & & & & \\
\hline
\end{tabular}

Fig. 1. Secretory leukocyte protease inhibitor (SLPI) primary structure. Identification of Pep4 sequence in SLPI structure (in grey).

place [10-13], leading to a protease-antiprotease imbalance. Under chronic inflammation, the imbalance is in favour of proteases, which widely overwhelm the inhibitory capacity of the endogenous proteinaceous inhibitors [5]. To reverse this protease-antiprotease imbalance and reinforce the elastase inhibitor's activity, different types of inhibitors and inhibitor formulations have been developed for wound dressings therapeutic applications. The design of synthetic low molecular weight, peptide-based [14-16] or non-peptide-based [17-19], elastase inhibitors has received considerable attention due to their potential therapeutic usefulness. The delivery of inhibitors or other active materials to the wound medium, promoted by wound dressings, can be accomplished by two different approaches: (i) the sequestration of elastase directly from the wound environment, with further protease inactivation or (ii) the direct release of the protease inhibitor into the wound medium, to further control the elastase levels in chronic non-healing wounds [4]. In previous studies [20,21], we have reported two different drug delivery systems, based on both wound dressing mechanisms described herein, to deliver novel elastase peptidic inhibitors to wound environment. The peptide sequence of these inhibitors, KRCCPDTCGIKCL (Pep4) and KRMMPDTMGIKML (Pep4M) [20], was selected from an endogenous elastase inhibitor, secretory leucocyte protease inhibitor (SLPI, Fig. 1). Pep4M is a mutation of Pep4 sequence, having replaced all cysteine residues by methionine residues. Both peptides revealed differential inhibitory activity towards elastase (porcine pancreatic elastase, PPE) when a specific residue (threonine, in this case) is in the phosphorylated and non-phosphorylated form [20]. The structure of peptides Pep4 and Pep4M and the influence of this posttranslational modification on their structure were evaluated herein by twodimensional NMR and molecular modelling studies. Additionally, these studies were also extended to another elastase inhibitorpeptide, PepBBI (WCTASVPPQCY), a synthetic peptide derived from the Bowman-Birk Inhibitor reactive loop [14,22-24], in order to establish a comparison between PepBBI and the previous peptides. Similarly to Pep4 and Pep4M, for PepBBI a distinct inhibitory behaviour was also observed relatively to elastase for the phosphorylated and non-phosphorylated version of the peptide (unpublished data), which is reflected in the structural conformations adopted by the peptide in solution.

\section{Materials and methods}

\subsection{NMR experiments}

\subsubsection{NMR sample preparation}

The elastase inhibitor-peptides Pep4 (KRCCPDTCGIKCL), Pep4M (KRMMPDTMGIKML) and PepBBI (WCTASVPPQCY) used in this study were custom-made by JPT Peptide Technologies GmbH (Berlin, DE) [25].
The NMR analysis of the tridecameric peptides (Pep4 and Pep4M) [20] and undecameric peptide (PepBBI) [14,24] was performed in aqueous solution $\left(90 \% \mathrm{H}_{2} \mathrm{O} / 10 \%{ }^{2} \mathrm{H}_{2} \mathrm{O}\right)$ at $283 \mathrm{~K}$ to slow down molecular tumbling and at $\mathrm{pH} 3.0$ to minimise the rate of exchange of $\mathrm{NH}$ protons with the solvent. Working at $\mathrm{pH}$ below 7 is standard for structural determination of proteins by NMR. The NMR samples were prepared at a concentration of approximately $4 \mathrm{mg} / \mathrm{mL}$ using water (with $10 \% \mathrm{D}_{2} \mathrm{O}$ ) as solvent, for signal lock.

\subsubsection{NMR spectra acquisition and analysis}

NMR data were acquired on a Bruker Avance II+ spectrometer operating at proton frequency of $500 \mathrm{MHz}$ (Fig. 2). Two-dimensional homonuclear (2D) NMR experiments were performed: a ${ }^{1} \mathrm{H}$-Doublequantum filtered COSY [26] with $2 \mathrm{k}$ points covering a spectral width of $6 \mathrm{kHz}$ and 512 increments in the indirect dimension; a TOCSY [27] with $60 \mathrm{~ms}$ of spin-lock time and a $2 \mathrm{k}$ points covering a spectral width of $6 \mathrm{kHz}$ and 512 increments in the indirect dimension and a NOESY $[28,29]$ with $300 \mathrm{~ms}$ mixing time and $2 \mathrm{k}$ points covering a spectral width of $6 \mathrm{kHz}$ and $1 \mathrm{k}$ increments in the indirect dimension. NOE buildup experiments were performed to confirm that with this mixing time the condition of initial buildup is still valid. In all cases suppression of the solvent signal was obtained using a Watergate sequence with gradients. Additional NOESY experiments were performed using $300 \mathrm{~ms}$ of mixing time in a Bruker Avance III NMR spectrometer, operating at $800 \mathrm{MHz}$ using a QXI probehead. 2k points covering a spectral width of $10.4 \mathrm{kHz}$ were measured for $1 \mathrm{k}$ increments in the indirect dimension, to obtain further structural constrains. The solvent signal was suppressed using excitation sculpting with gradients. The Topspin software was used to process the data. All experiments were zero filled to duplicate the number of points in both dimensions. NOESY and TOCSY data were multiplied in both dimensions by a cosine squared windowing function prior to Fourier transform. COSY data were multiplied in both dimensions by a sine squared function prior to Fourier transform. Baseline correction was made following Fourier transformation using a 5th degree polynomial function. Peak lists of the three aforementioned 2D spectra were generated by interactive peak picking with further automatic integration of the peak volumes in the SPARKY software [30] (Table SI1-3). Peak assignments in COSY, TOCSY and NOESY spectra were performed using the sequential assignment method described by Wüthrich $[31,32]$. The chemical shifts and volumes of the NOESY crosspeaks were converted into XEASY format and input in the CYANA 2.0 program for structure calculations and analysis.

\subsubsection{Structure determination}

Assignments of ambiguous NOESY cross peaks were obtained by using the module CANDID in the program CYANA [33]. A total of 100 conformers were generated by CYANA and the 20 conformers with lowest target function were used to represent the 3D NMR structural bundle (Table 1 ). The quality of the structures was 

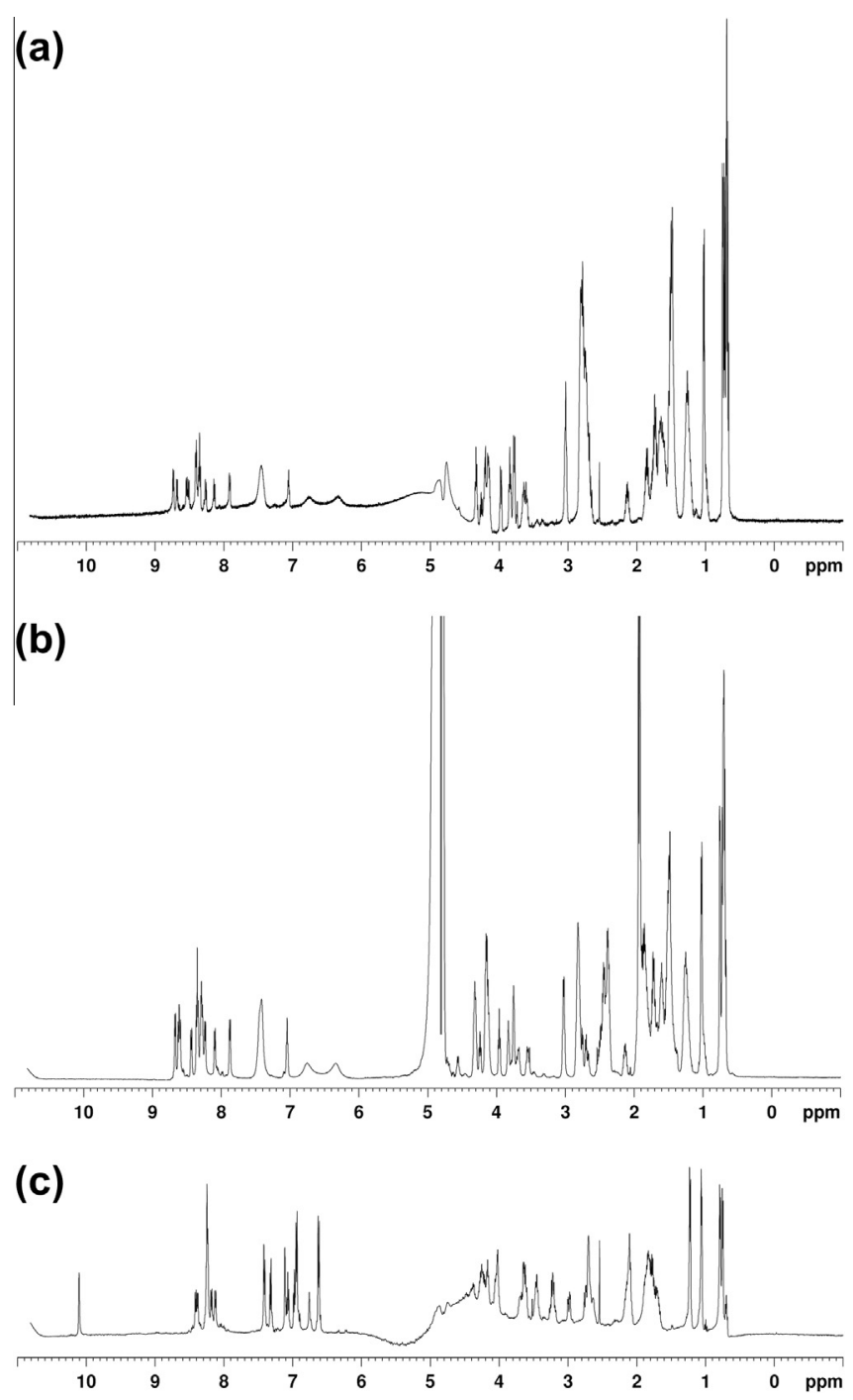

Fig. 2. Pep4 (a), Pep4M (b) and PepBBI (c) inhibitor-peptides proton NMR spectra $\left({ }^{1} \mathrm{H}\right.$ NMR). These spectra were acquired in a $500 \mathrm{MHz}$ Bruker NMR spectrometer.

evaluated through Ramachandran plots (Table 1), obtained using PROCHECK-NMR program $[34,35]$. The final structures were displayed and analysed on MOLMOL [36] and PyMOL [37] software, respectively.

\subsection{Molecular docking assays}

Molecular docking studies were performed using the X-ray structure of PPE with PDB code 1B0E with Autodock/Vina [38]. The grid for the ligand conformational search calculations was placed with its centre located at the PPE binding site. The docking grid size was $32 \times 59 \times 27$ grid points with $1.0 \AA$ spacing. Conformational search was done using the Iterative Local Search algorithm and 10 conformational poses with the lowest binding free energy were recorded. The peptides were modelled as fully flexible except for the main-chain peptide amide bonds. Peptides were docked in their phosphorylated and non-phosphorylated form: Pep4 and Pep4(P) phosphorylated on Thr7; Pep4M and Pep4M(P) phosphorylated on Thr7; PepBBI and PepBBI(P) phosphorylated on Thr3, Ser5 and both residues. The best conformations with the lowest binding free energy were selected and analysed.
Table 1

Characterisation of the 20 energy-refined CYANA conformers representing the NMR structures of Pep4, Pep4M and PepBBI.

\begin{tabular}{|c|c|c|c|}
\hline Parameters & Pep4 ${ }^{\mathrm{a}}$ & Pep4M & PepBBI $^{\mathrm{a}}$ \\
\hline \multicolumn{4}{|c|}{ Restrains used in the structure calculations } \\
\hline \multicolumn{4}{|l|}{ Number of distance restrains } \\
\hline Intra $(|i-j|=0)$ & $\begin{array}{l}81 \\
(61.8 \%)\end{array}$ & $\begin{array}{l}105 \\
(65.6 \%)\end{array}$ & $\begin{array}{l}72 \\
(66.1 \%)\end{array}$ \\
\hline Short-range $(|i-j|=1)$ & $\begin{array}{l}50 \\
(38.2 \%)\end{array}$ & $\begin{array}{l}49 \\
(30.6 \%)\end{array}$ & $\begin{array}{l}36 \\
(33.0 \%)\end{array}$ \\
\hline Medium-range $(1<|i-j| \leqslant 4)$ & $\begin{array}{l}0 \\
(0 \%)\end{array}$ & $\begin{array}{l}6 \\
(3.8 \%)\end{array}$ & $\begin{array}{l}1 \\
(0.9 \%)\end{array}$ \\
\hline Long-range $(|i-j|>4)$ & 0 & 0 & 0 \\
\hline Total & $\begin{array}{l}131 \\
(100 \%)\end{array}$ & $\begin{array}{l}160 \\
(100 \%)\end{array}$ & $\begin{array}{l}109 \\
(100 \%)\end{array}$ \\
\hline \multicolumn{4}{|l|}{ Number of torsion angle restrains } \\
\hline$\Phi$ & 0 & 0 & 0 \\
\hline \multicolumn{4}{|l|}{ Final CYANA structures } \\
\hline Target function $\left(\AA^{\mathrm{b}}\right)$ & $0.13 \pm 0.02$ & $0.038 \pm 0.008$ & $0.04 \pm 0.02$ \\
\hline \multicolumn{4}{|l|}{ Structural statistics } \\
\hline \multicolumn{4}{|c|}{ Atomic root mean square deviation (RMSD) from the mean structure $(\AA)$} \\
\hline Backbone atoms $(\mathrm{N}, \mathrm{C} \alpha, \mathrm{C}, \mathrm{O})$ & $2.3 \pm 0.5$ & $1.8 \pm 0.6$ & $2.0 \pm 0.6$ \\
\hline All heavy atoms & $3.4 \pm 0.7$ & $2.9 \pm 0.7$ & $3.4 \pm 0.7$ \\
\hline \multicolumn{4}{|l|}{ Ramachandran analysis ${ }^{\mathrm{b}}$} \\
\hline Most favoured regions (\%) & 63.9 & 50.0 & 43.6 \\
\hline Additional allowed regions (\%) & 28.9 & 48.9 & 50.7 \\
\hline Generously allowed regions (\%) & 7.2 & 1.1 & 5.7 \\
\hline Disallowed regions (\%) & 0 & 0 & 0 \\
\hline
\end{tabular}

a Peptide structures generated without constrains on disulphide bridges.

b Structure quality was analysed by using PROCHECK [34].

\section{Results and discussion}

\subsection{NMR studies}

The NMR structure of the peptides, Pep4, Pep4M and PepBBI, were determined by two-dimensional homonuclear experiments, COSY and TOCSY, for spin-system assignment and NOESY experiments for sequential assignment of the spin-systems and distance restraints (see materials and methods section, Table 1 and Fig. 3). Data were collected at $\mathrm{pH}$ below 7 to minimise the proton exchange of the $\mathrm{NH}$ groups with the solvent. Preliminary data collected at $500 \mathrm{MHz}$ showed no significant differences in the intensity or number of NOESY cross peaks between $\mathrm{pH} 7$ and 3 in the aliphatic region of the spectrum. This is the region where the amino acid side chains display signals and where contacts between amino-acids resulting from different structures should be revealed. At lower $\mathrm{pH}$ the $\mathrm{NH}$ region has better spectral characteristics and since only the aspartate side chain of Pep4 and Pep4M is expected to titrate in this $\mathrm{pH}$ range, a low $\mathrm{pH}$ was selected for data collection. These peptides have a size that places them near the condition where the correlation time is close to the reciprocal of the Larmor frequency and NOE effect is eliminated. To minimise this effect, the experimental temperature was reduced to $283 \mathrm{~K}$, which led to the observation of clear intra-residue cross-peaks. In this context the low abundance of medium range, and total lack of long-range cross peaks discussed below are clear indications of substantial conformational mobility of these peptides in solution. This experimental observation agrees with the predictions of $\operatorname{DisEMBL}(1.5)$ computational tool [39] that indicate a large probability of these peptides being disordered. NOESY data were sufficient to observe proton-proton NOEs between the amide proton of one residue and the $\alpha$-proton of the preceding residue, for all residues in these three peptides (Fig. 3). In Pep4 NOESY spectrum, no long or even medium range NOE interactions occur, only short-range interactions, such as $\mathrm{HN}-\mathrm{HN}, \mathrm{H} \alpha-\mathrm{HN}, \mathrm{H} \beta-\mathrm{HN}$, were observed. 


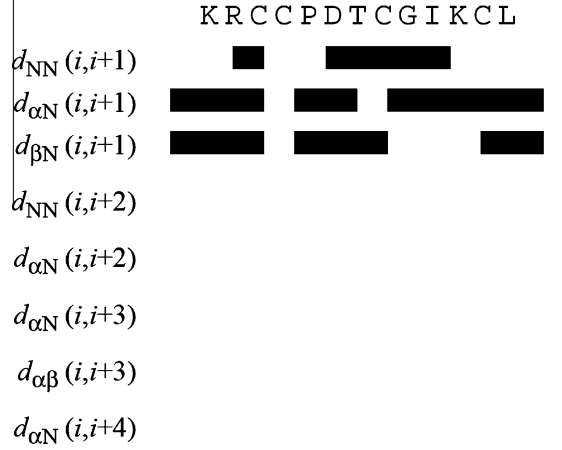

10

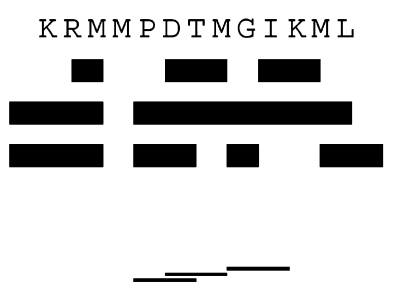

10

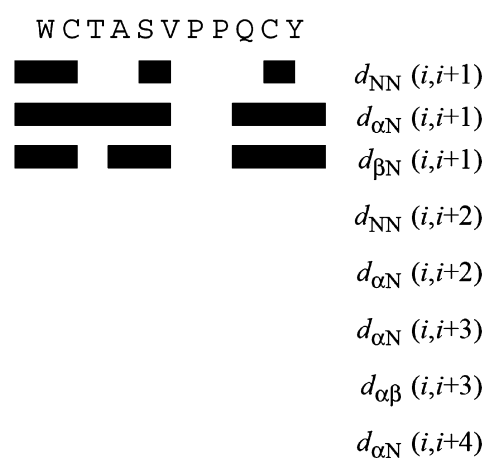

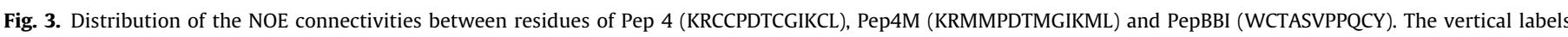

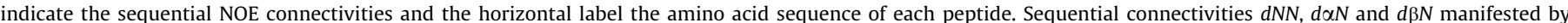

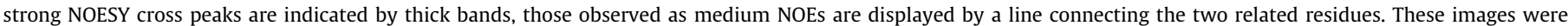
generated using the CYANA software [33].

In contrast to the previous peptide, for Pep4M the NOESY spectrum revealed the existence of medium range NOE connectivities, between $\mathrm{H \alpha}_{\mathrm{i}}-\mathrm{NH}_{\mathrm{i}+2}$ protons, for $\mathrm{Pro}^{5}-\mathrm{Thr}^{7}$, Asp ${ }^{6}-\mathrm{Met}^{8}$, $\mathrm{Met}^{8}-\mathrm{Ile}^{10}$.

PepBBI, also shows medium range NOEs, in this case between $\mathrm{H} \beta_{\mathrm{i}}-\mathrm{NH}_{\mathrm{i}+2}$ protons for Pro $^{8}-\mathrm{Cys}^{10}$, although this data is not shown in Fig. 3 (see Table 1). In PepBBI, as for the other two peptides, no long range NOEs were found.

These data allow us to conclude that Pep4M has a lower degree of conformational flexibility in solution than Pep4 and PepBBI, which leads to the observation of more medium range distance

\section{(a)}

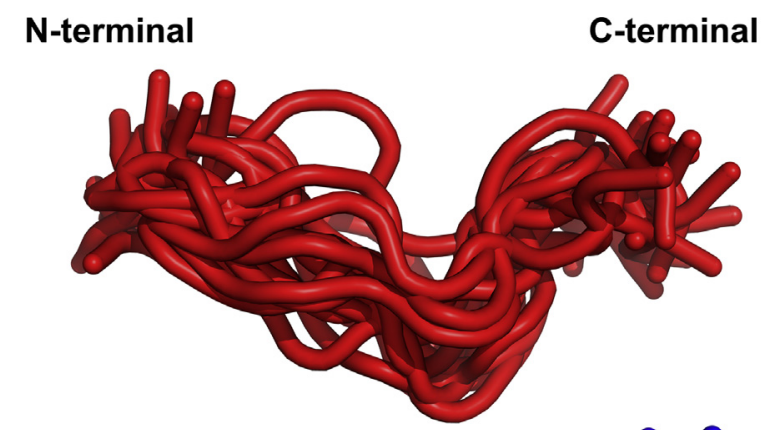

(b)

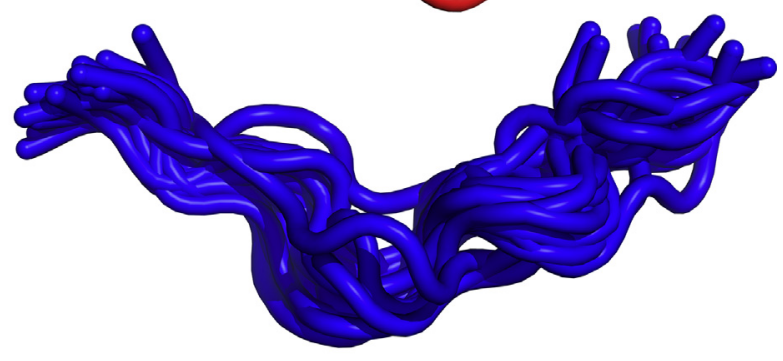

(c)

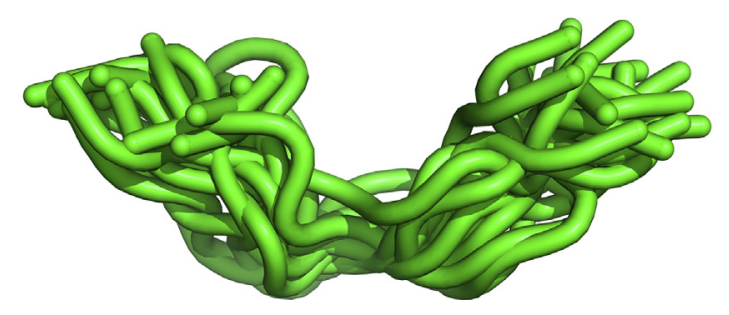

Fig. 4. Comparison of three-dimension structures obtain by 2D-NMR for Pep4, Pep4M and PepBBI. Bundles of the 20 energy-minimised conformers were used to represent the NMR structure of the inhibitor-peptides: Pep4 (a); Pep4M (b) and PepBBI (c). Figures a-c were prepared by using the program MOLMOL [36] and PyMOL [37]. restraints. Data reported in Table 1 corroborate the previous statements. In the NOESY spectra of these three peptides $62-66 \%$ corresponds to intra-residue interactions, $31-38 \%$ to short-range interactions and Pep4M and PepBBI shown almost 4\% and 1\% of medium-range interactions, respectively.

The final set of 20 structures for Pep4M has a mean global backbone R.M.S.D. of $1.8 \pm 0.6 \AA$ (Table 1 ) slightly lower than those obtained for Pep4 and PepBBI, $2.3 \pm 0.5$ and $2.0 \pm 0.6 \AA$, respectively. These RMSD values are well in line with values reported in the literature for peptides of similar size $[40,41]$. The final Pep4M and PepBBI families (after restrained energy minimisation) have an average total target function of $0.038 \pm 0.008$ and $0.04 \pm 0.02 \AA^{2}$ (CYANA units), respectively, while the corresponding value for Pep4 is $0.13 \pm 0.02 \AA^{2}$. Low target function values have been achieved for the conformer families of these peptides, although for Pep4 this value is almost ten times higher than those observed for the other peptides. This might be due to the fact that no medium range connectivities were observed in the NOESY spectrum of Pep4, reflecting a higher degree of conformational flexibility in the bundle of 20 lowest energy conformers.

The Ramachandran plots obtained for the geometry validation show that $92.8 \%, 98.9 \%$ and $94.3 \%$ of the residues of Pep4, Pep4M and PepBBI, respectively, are in the most favoured and allowed regions (Table 1 ). None of the structures have residues in the disallowed region.

\subsubsection{Peptides three dimension structure comparison}

The backbone of Pep4M and PepBBI is well defined and conserved along the 13 and 11 amino acid residues, respectively (with

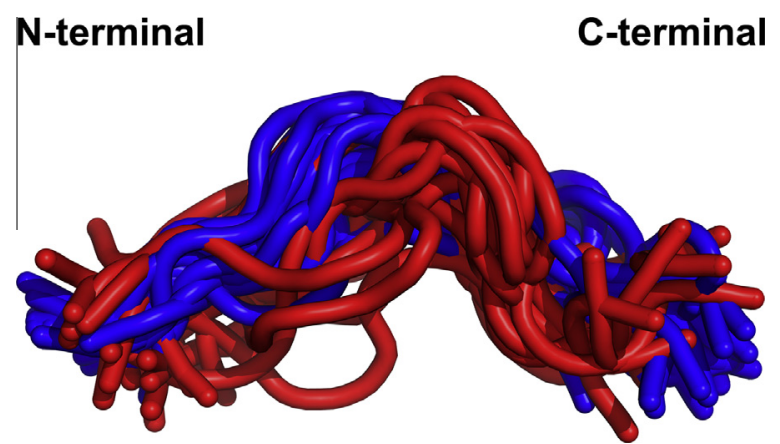

Fig. 5. Structural comparison of Pep4 (red structure) and Pep4M (blue structure) The calculated 20 conformers of Pep4 are superimposed with the conformers of Pep4M. (For interpretation of the references to colour in this figure legend, the reader is referred to the web version of this article.) 
(a) N-terminal

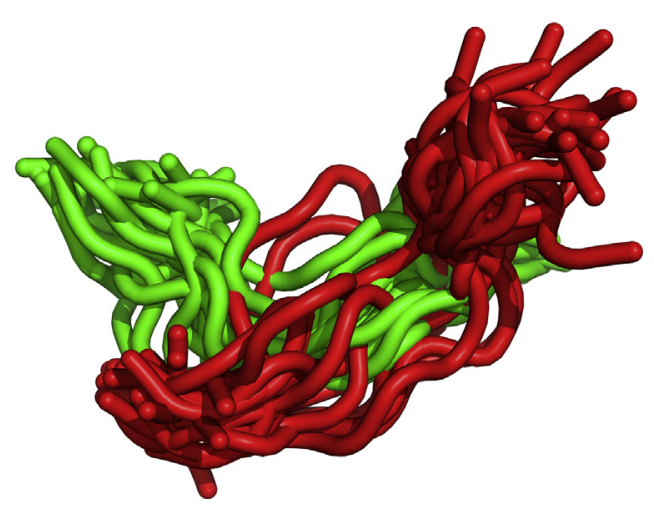

(b) N-terminal

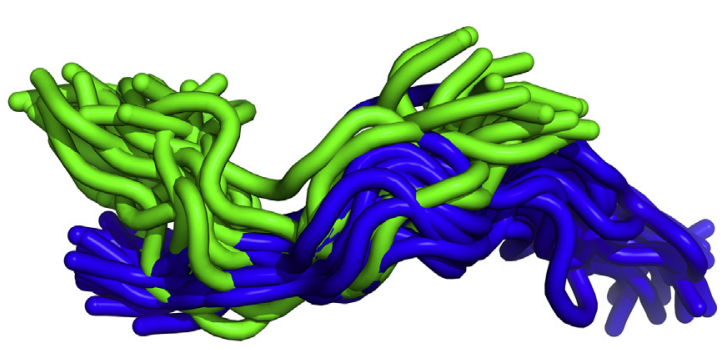

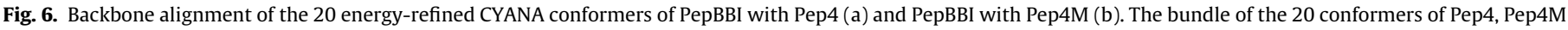

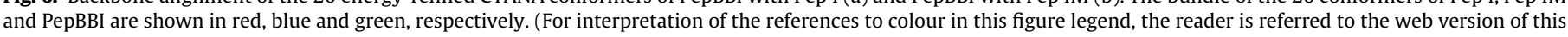
article.)

(a) N-terminal

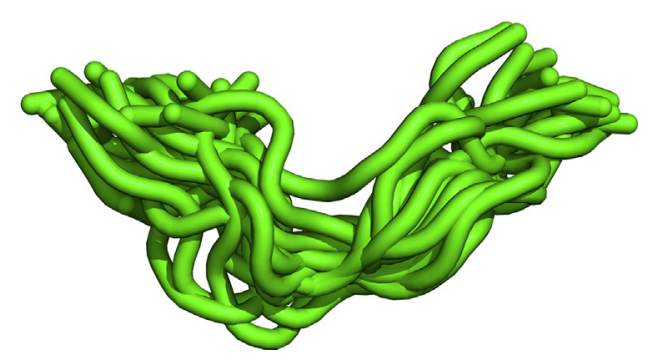

(b)

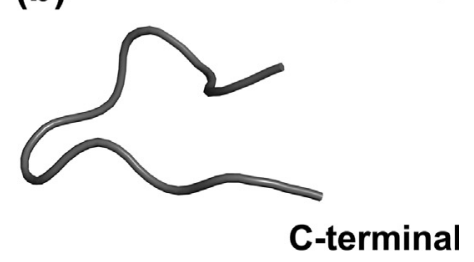

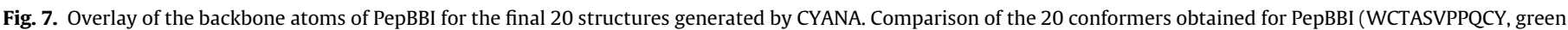

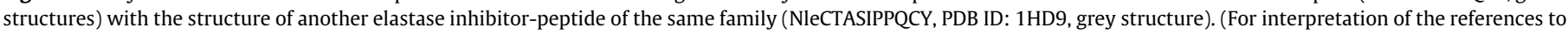
colour in this figure legend, the reader is referred to the web version of this article.)

the exception of two conformers, Fig. $4 \mathrm{~b}$ and c). However, the same behaviour was not observed for the other peptide, Pep4 (Fig. 4a), in which only 14 of the 20 energy-minimised conformers have the backbone completely superimposed.

The comparison of Pep4 and Pep4M final structures reveal several structural differences. These peptides have the same primary structure, although with the mutation of four cysteine residues by four methionine residues. The presence of methionine residues, which has a longer side chain, induces steric hindrance that is reflected in the observed structural changes. This structural change inputs a considerable difference in the three dimensional (3D) structures of these peptides (Fig. 5a and b). Fig. 5 shows the alignment of the bundles of 20 conformers obtained for both peptides. In the alignment of Pep4 to Pep4M final conformers, it is possible to observe that Pep4 structure (red structure) seems to be shifted right comparatively to Pep4M (blue structure).

Comparing the 20 energy refined conformers obtained for Pep4 and PepBBI (Fig. 6a), it is thus possible to state that Pep4 only aligns with PepBBI in three residues, $\mathrm{Pro}^{5}, \mathrm{Asp}^{6}$ and $\mathrm{Thr}^{7}$ with Pro $^{7}$, Pro ${ }^{8}$ and $\mathrm{Gln}^{9}$, respectively. Even so, few of the 20 conformers obtained for both peptides show this alignment. Therefore, poor alignment was observed between peptides Pep4 and PepBBI.

The overlay of the backbone atoms of Pep4M and PepBBI for the final 20 structures generated by CYANA (Fig. 6b), revealed the alignment of seven amino acid residues of these peptides, $\mathrm{Met}^{3}$ Gly ${ }^{9}$ with $\mathrm{Ser}^{5}-\mathrm{Tyr}^{11}$, respectively. In this case, a good alignment between Pep4M and PepBBI was achieved comparatively to Pep4 and PepBBI superposition.

Fig. 7 shows a comparison of PepBBI (WCTASVPPQCY) bundle of 20 conformers with the structure of another peptide from the same family (NleCTASIPPQCY, PDB ID:1HD9) [23]. These undecameric peptides possess the same primary structure, with only two mutations in the first and in the sixth amino acid residue $(\mathrm{W} \rightarrow$ Nle and $\mathrm{V} \rightarrow \mathrm{I}$ ). According to literature [14,24], these small modifications in the PepBBI structure induces considerable enhancement in the inhibitor constant $\left(K_{\mathrm{i}}\right)$ values ( $K_{\mathrm{i}}$ decreases an order of magnitude). The amino acid sequence of these peptides was designed based on the reactive-site loop of Bowman-Birk inhibitor (BBI), a protease inhibitor protein. The Bowman-Birk inhibitor interacts with serine proteases, such as elastase, in an extended or "canonical" loop $[14,24]$. This loop, is frequently constrained by the presence of disulphide bridges and/or extensive hydrogen-bonding networks [14]. Peptide NleCTASIPPQCY is found to retain essentially the same three-dimension arrangement of both backbone and side chains as observed in BBI complete protein (structure obtained at $\mathrm{pH}$ 3.8). Nevertheless, PepBBI (WCTASVPPQCY) does not retain the loop shape characteristic of the Bowman-Birk inhibitor (Fig. 7). In PepBBI, no constrains were observed due to disulphide bridges and therefore instead of a "canonical" loop a "W" shape was attained (structure obtained at $\mathrm{pH}$ 3.0).

\subsection{Molecular modelling studies}

The posttranslational modification (phosphorylation) of these tridecameric and undecameric peptides induced a significant decrease in their inhibitory activity towards elastase. Nevertheless, this effect was more accentuated in the peptides Pep4 and Pep4M [20] than in peptide PepBBI (unpublished data). Consequently, this fact is reflected in the values of binding free energy (Table 2) for the interactions enzyme-peptide, in the phosphorylated and 
Table 2

Summary of the binding free energies involved in the interaction of the nonphosphorylated and phosphorylated peptides (Pep4, Pep4M and PepBBI) with elastase.

\begin{tabular}{lllc}
\hline \multirow{2}{*}{ Peptides } & $\Delta G(\mathrm{kcal} / \mathrm{mol})$ & & \\
\cline { 2 - 4 } & Non-phosphorylated & Phosphorylated & [Phos-(Non-Phos)] \\
\hline Pep4 & -6.0 & $-5.2(\mathrm{Thr})$ & 0.8 \\
Pep4M & -5.8 & $-4.8(\mathrm{Thr})$ & 1.0 \\
PepBBI & -7.0 & $-7.1(\mathrm{Ser})$ & -0.1 \\
& & $-6.8(\mathrm{Thr})$ & 0.2 \\
& & $-7.4($ Ser, Thr) & -0.4 \\
\hline
\end{tabular}

non-phosphorylated format. Thus, for Pep4 and Pep4M higher energy differences were achieved $(\sim 1 \mathrm{kcal} / \mathrm{mol})$ comparatively to PepBBI (lower than 0.2).

To evaluate the structural modifications that prompted such alteration on the inhibitory capacity of these peptides relatively to elastase (PPE), a combined study involving two dimensional NMR studies and molecular docking simulations was performed for the three peptides involved in this study. Therefore, the 20 energy-minimised conformers obtained by 2D-NMR for Pep4, Pep4M and PepBBI (shown in previous section), were used to establish interactions between these peptides and active centre of the serine protease elastase. Rigid docking experiments were performed using the NMR energy-minimised structures, however, as expected, the solution structure of the three peptides by NMR did not allow an appropriate interaction with the active centre of the enzyme. This is justified by the lack of peptide conformational freedom to adapt to the binding site. This fact, prompted us to do flexible docking experiments in order to model in a more realistic way the binding of the peptides against elastase.

The Pep4 and Pep4M lowest energy structures were docked (using a fully flexible model of the peptides) towards the active centre of elastase (PPE, Figs. 8 and 9). The fully flexible docking experiments show that, both peptides display an extended conformation along the crevice formed between the two elastase domains (Figs. 8a and 9a). In contrast the phosphorylated form of Pep4 and Pep4M peptides adopt a hairpin conformation and these structures are confined to the elastase active centre region (Figs. 8b and 9b). The posttranslational modification (phosphorylation) of the threonine residue in Pep4 and Pep4M induces the formation of a large bent conformation, mainly due to electrostatic and inter-residue interactions (Fig. 10). In Pep4(P), the phosphothreonine $\operatorname{Thr}(\mathrm{P}) 7$ is able to establish electrostatic interactions with the guanidinium group of Arg2 and the $\mathrm{NH}_{\varepsilon}$ group of Lys1 (Fig. 10a) and hydrogen bonds with the amine group of Lys1 (Fig. 10b). In Pep4M(P), the phosphate group of $\mathrm{Thr}(\mathrm{P}) 7$ establish electrostatic interactions with the positively charged residue Arg2 (Fig. 10d). Additionally, in this peptide it is also possible to observe hydrogen bonds between alternate residues, such as Thr(P)7-Gly9 and Asp6-Met8 (Fig. 10c). Differential binding energies were registered for the peptides Pep4 and Pep4M phosphorylated and non-phosphorylated when docked to PPE (Table 2). Thus, the inter-residue interactions prompted by the phosphorylated residue $\operatorname{Thr}(\mathrm{P}) 7$, decreases the peptide-enzyme interactions. This is in accordance with previous studies [20], which reported a decrease in the inhibitory activity of peptides Pep4 and
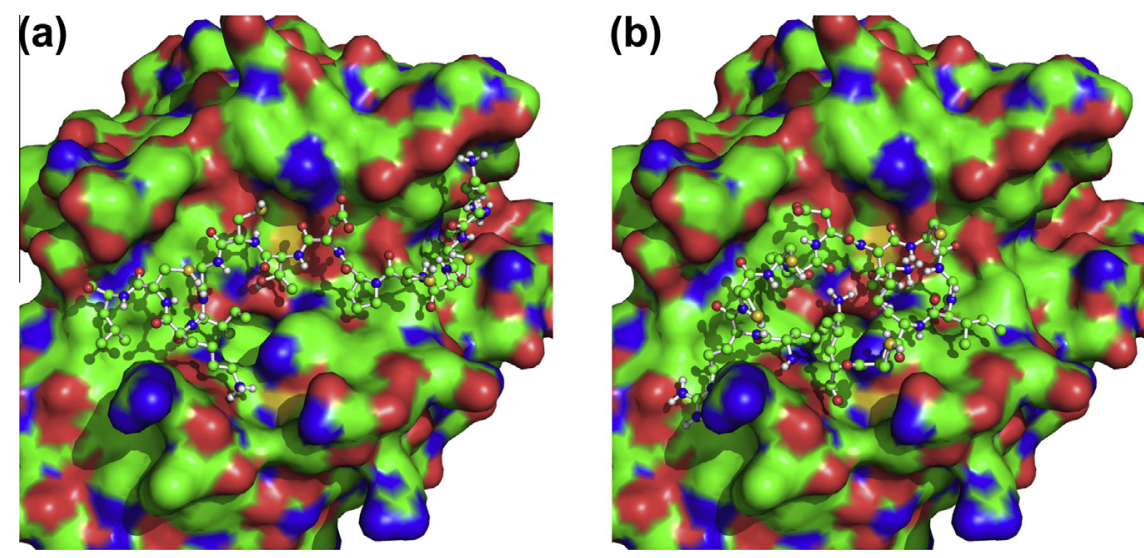

Fig. 8. Docking complexes with the highest binding free energy of Pep4 to PPE, in the non-phosphorylated (a) and phosphorylated (b) form.
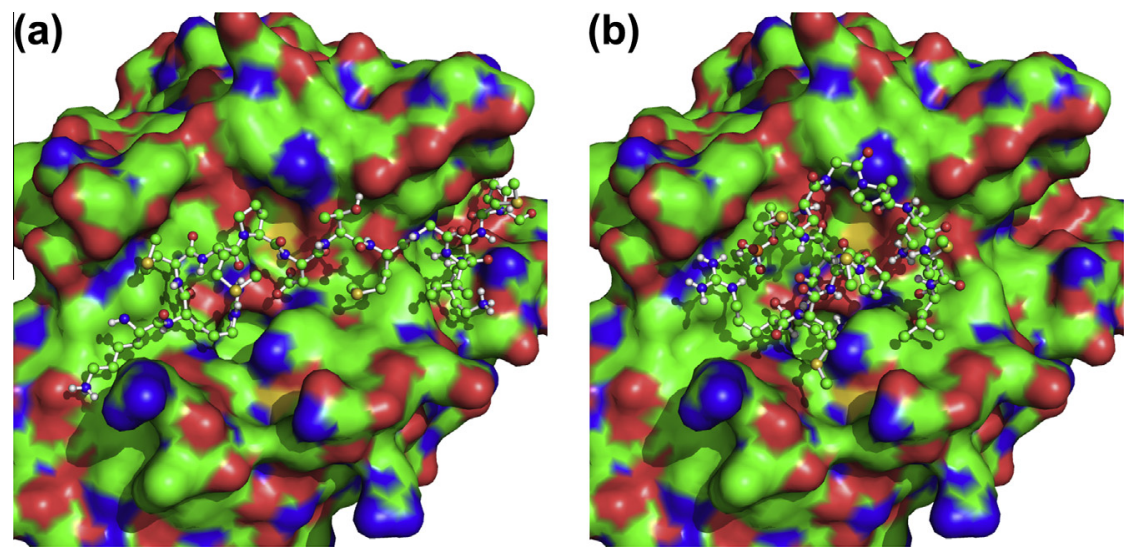

Fig. 9. Docking complexes with the highest binding free energy of Pep4M to PPE, with the peptide non-phosphorylated (a) and phosphorylated (b). 


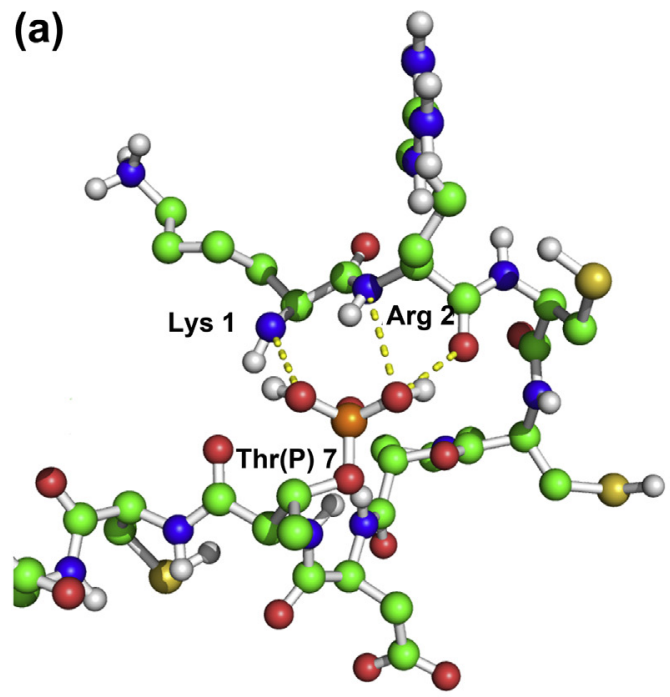

(b)

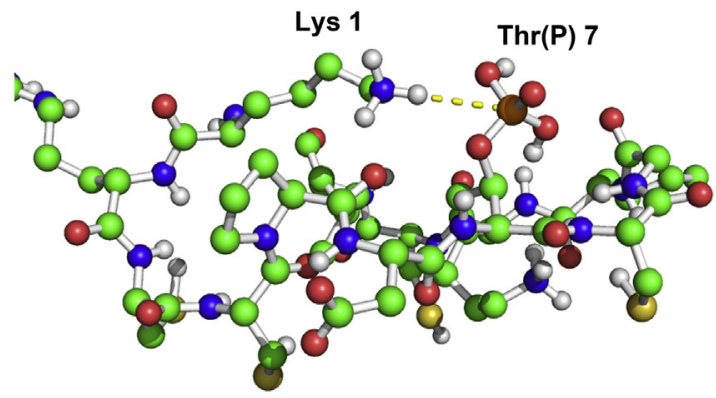

(c)

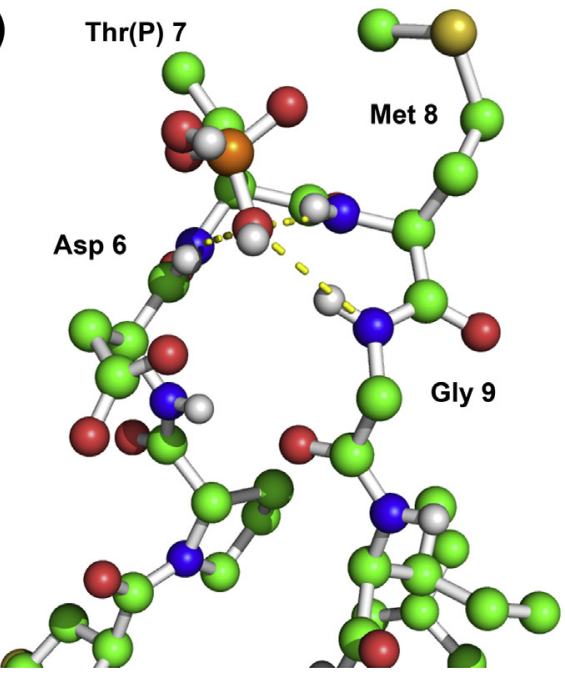

(d)

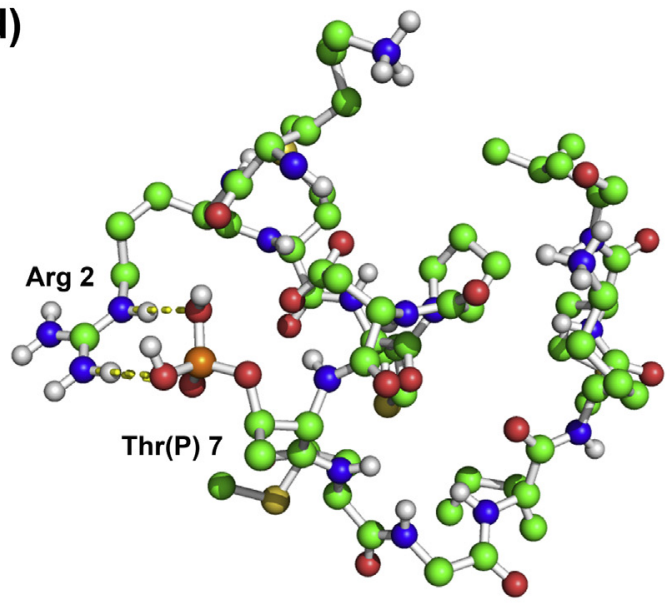

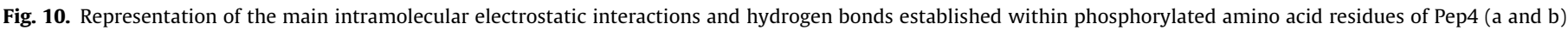
and Pep4M (c and d).

Pep4M towards PPE, for the phosphorylated version relatively to the non-phosphorylated form.

Regarding to PepBBI, a Bowman-Birk inhibitor based peptide, a distinct inhibitory behaviour relatively to PPE is also evident, when it is in the phosphorylated and non-phosphorylated form (unpublished data). Contrarily to the previous peptides, PepBBI possess three potential phosphorylation sites: Thr3; Ser5 and Tyr11. However, as the protein kinase used to perform the phosphorylation of the current peptide was a serine/threonine kinase (Casein Kinase I $\delta$ ), it is thus expected that the phosphorylation only occurs at the Thr3, Ser5 or at both residues (Fig. 11). Similarly to Pep4 and Pep4M, PepBBI displays an extended conformation in between the two domains of elastase (Fig. 11a). Considering that the phosphorylation only occurs at Thr3 residue, PepBBI adopts the shape of a large loop, with the phosphate group pointing out of the structure (Fig. 11b). This structural modification is prompted by electrostatic interactions and hydrogen bonds between the phosphothreonine residue $\operatorname{Thr}(\mathrm{P}) 3$ and the side chain of His57 at the catalytic triad of elastase (Fig. 12a). Additionally, also occur hydrogen bonds are formed between the phosphate group of $\operatorname{Thr}(\mathrm{P}) 3$ and Asp60 residue of the protease (Fig. 12a). These interactions may reduce the activity of elastase, although to a less extent than that registered in the non-phosphorylated version of the peptide. If the phosphorylation took place at Ser5 residue, this process does not induce any relevant structural differences relatively to the non-phosphorylate peptide (Fig. 11a and c). This is reflected in the binding energy for the peptide-enzyme interaction that remains unaltered, whether the PepBBI is phosphorylated $(-7.1 \mathrm{kcal} / \mathrm{mol})$ or non-phosphorylated $(-7.0 \mathrm{kcal} / \mathrm{mol}$, Table 2$)$. Thus, these data do not corroborate the experimental data that stress distinct inhibitory capacity for the phosphorylated and non-phosphorylated versions of the peptide. Therefore, it is not expected that the phosphorylation of PepBBI occurs at Ser5 residue. The third option, in which both residues might be phosphorylated, the peptide maintains an extended conformation having both phosphate groups inserted in the elastase active site (Fig 11d). According to literature, it is possible to phosphorylate alternated residues, even if small amino acid residues such as alanine or glycine occur in between them [42], and also adjacent residues $[43,44]$. The phosphate group of the phosphothreonine establish hydrogen bonds with hydroxyl group of Ser195 at elastase catalytic triad (Fig. 12b). Similarly to the first option of phosphorylation site $(\operatorname{Thr}(\mathrm{P}) 3)$, this interaction may cause an impediment to the normal elastase activity and consequently a decrease in its activity. 

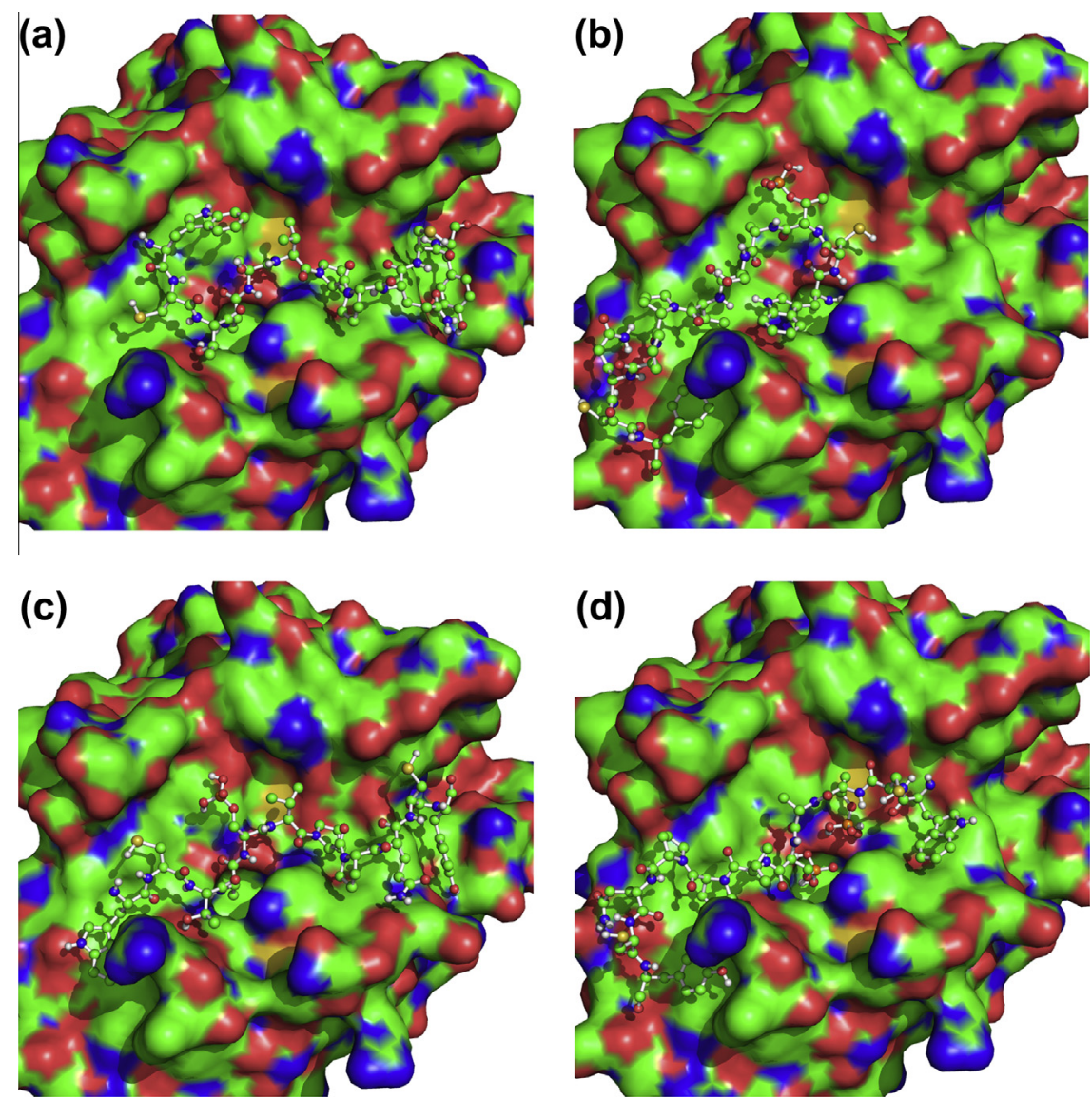

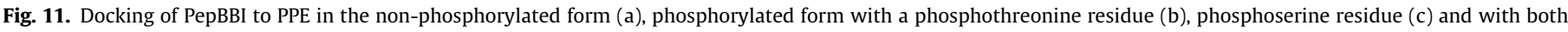
phosphoresidues (d).

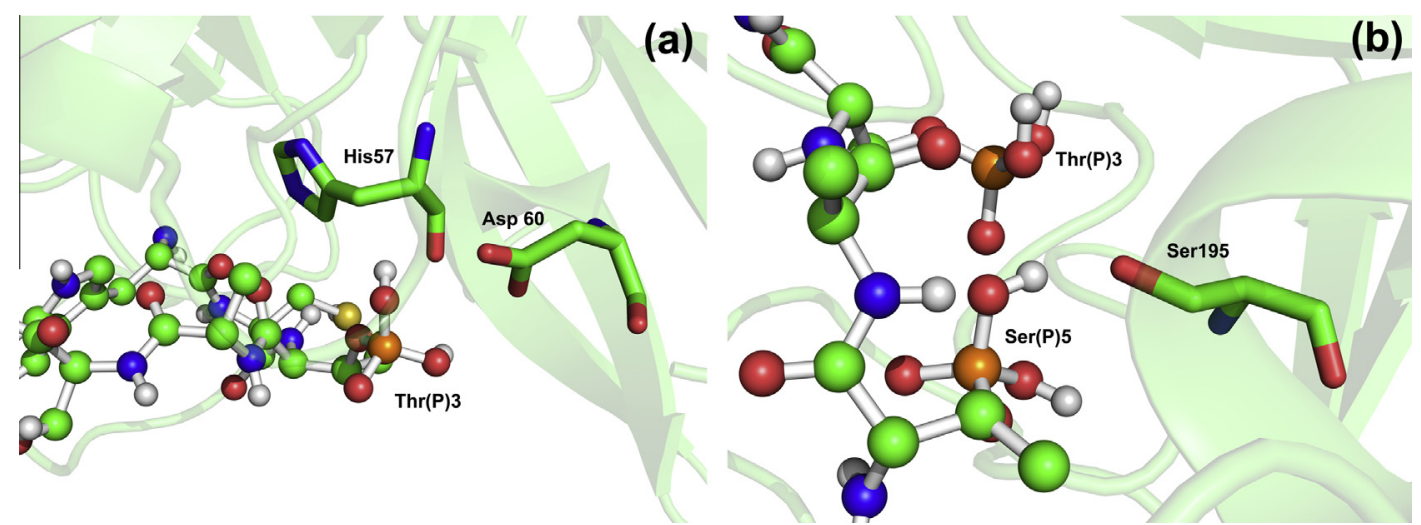

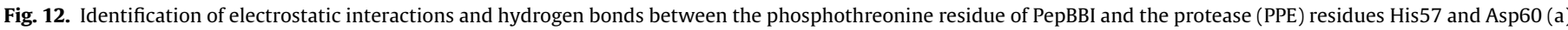
and Ser195 (b).

Nevertheless, this reduction in elastase activity is less pronounced than in the non-phosphorylated version of the peptide, according to the experimental data obtained for the phosphorylation of Pep$\mathrm{BBI}$ in solution (unpublished data).

Therefore, considering the three possibilities of potential phosphorylation sites in PepBBI, the options that mostly corroborates with the experimental data is the first and third option, in which the phosphorylation occurs at Thr3 or at Thr3 and Ser5 residue simultaneously. Although, serine residues rather than threonine residues appeared to be more preferred phosphorylation targets $[43,45,46]$, in this case the opposite seemed to occur.
Hence, the phosphorylation in Pep4 and Pep4M occur at the phosphoresidue Thr7 and in PepBBI all evidences point out to the phosphorylation occurring at the phosphoresidue Thr3 or Thr3 and Ser5 jointly. The phosphorylation of these residues in all the three peptides induced a conformation alteration (formation of a large loop, except when in presence of a pair of phosphoresidues), similar to what has been reported in other peptides $[47,48]$.

\section{Conclusions}

The combined approach of two-dimensional NMR and molecular docking techniques allowed the detection of differential 
conformations adopted by the tridecameric (Pep4 and Pep4M) and undecameric (PepBBI) peptides in the phosphorylated and nonphosphorylated form. This distinct conformation acquired by the three peptides, is compatible with the differential inhibitory activity observed for these peptides towards elastase (PPE) in previous studies [20].

The 2D-NMR structural studies on the peptides Pep4, Pep4M and PepBBI, revealed significant structural flexibility in all the peptides, when present in solution. Nevertheless, the final set of 20 energy-minimised conformers obtained for these peptides revealed a good backbone overlay, especially in the case of Pep4M where the presence of several medium range interactions $(1<|i-j| \leqslant 4)$, indicate lower conformational mobility of the peptide in solution.

The molecular docking studies demonstrated the distinct conformations acquired by these peptides when their potential phosphorylation sites (threonine) carry or not a phosphate group in their structure. Additionally, these studies also highlighted the inter-residue and inter-molecular interactions (electrostatic interactions and hydrogen bonds) that prompted these structural modifications. The differences between the solution and docked structures show that these peptides behave as the very important class of intrinsically disordered proteins, which only assume the active conformation upon meeting their physiological partners.

Phosphorylation process seemed to play a determinant role in the structure and in the biological properties of the three peptides included in this study. These results are important for the incorporation and choice of these peptides for wound dressings described in other works of our group [20,21,49-51].

\section{Acknowledgments}

The authors gratefully acknowledge the financial support of the Portuguese Foundation for Science and Technology (scholarship SFRH/BD/36522/2007 and PEst-OE/EQB/LA0004/2011), FEDER (European Fund for Regional Development)-COMPETE-QREN-EU and the European Project Lidwine - Multifunctional medical textiles for wound (e.g. Decubitus). We acknowledge CERMAX at ITQB-UNL and Rede Nacional de RMN for access to the facilities. Rede Nacional de RMN is supported with funds from FCT, Projecto de Re-equipamento Científico contract REDE/1517/RMN/2005, Portugal. Micaêlo, N.M. acknowledges the contract research program "Compromisso com a Ciência" reference: C2008-UMINHO-CQ-03 and access to the Minho University GRIUM cluster.

\section{Appendix A. Supplementary material}

Supplementary data associated with this article can be found, in the online version, at http://dx.doi.org/10.1016/j.reactfunctpolym. 2013.02.011.

\section{References}

[1] B. Cullen, R. Smith, E. McCulloch, D. Silcock, L. Morrison, Wound Repair and Regeneration 10 (2002) 16-25.

[2] B. Cullen, P.W. Watt, C. Lundqvist, D. Silcock, R.J. Schmidt, D. Bogan, N.D. Light, The International Journal of Biochemistry and Cell Biology 34 (2002) 15441556

[3] N.B. Menke, K.R. Ward, T.M. Witten, D.G. Bonchev, R.F. Diegelmann, Clinics in Dermatology 25 (2007) 19-25.

[4] J.V. Edwards, P. Howley, R. Davis, A. Mashchak, S.C. Goheen, International Journal of Pharmaceutics 340 (2007) 42-51.

[5] M.-L. Zani, S.M. Nobar, S.A. Lacour, S. Lemoine, C. Boudier, J.G. Bieth, T. Moreau, European Journal of Biochemistry 271 (2004) 2370-2378.

[6] J.V. Edwards, S.H. Phyllis, Journal of Biomedical Materials Research Part A 83A (2007) 446-454.

[7] M.-L. Zani, K. Baranger, N. Guyot, S. Dallet-Choisy, T. Moreau, Protein Science 18 (2009) 579-594.
[8] T.V. Tikhonova, I.P. Gladysheva, N.I. Larionova, FEBS Letters 362 (1995) 225228.

[9] R. Alasbahi, M. Melzig, Scientia Pharmaceutica 76 (2008) 471-483.

[10] S.M. Nobar, M.-L. Zani, C. Boudier, T. Moreau, J.G. Bieth, FEBS Journal 272 (2005) 5883-5893.

[11] C. Boudier, J.G. Bieth, Biochem J 303 (Pt 1) (1994) 61-68.

[12] P.J. Ossanna, S.T. Test, N.R. Matheson, S. Regiani, S.J. Weiss, The Journal of Clinical Investigation 77 (1986) 1939-1951.

[13] D. Johnson, J. Travis, Journal of Biological Chemistry 254 (1979) 4022-4026.

[14] J.D. McBride, E.M. Watson, A.B.E. Brauer, A.M. Jaulent, R.J. Leatherbarrow, Peptide Science 66 (2002) 79-92.

[15] J.D. McBride, R.J. Leatherbarrow, Current Medicinal Chemistry 8 (2001) 909917.

[16] P.A. Wright, R.C. Wilmouth, I.J. Clifton, C.J. Schofield, European Journal of Biochemistry 268 (2001) 2969-2974.

[17] H. Ohbayashi, Expert Opinion on Therapeutic Patents 15 (2005) 759-771.

[18] R.J. Cregge, S.L. Durham, R.A. Farr, S.L. Gallion, C.M. Hare, R.V. Hoffman, M.J. Janusz, H.-O. Kim, J.R. Koehl, S. Mehdi, W.A. Metz, N.P. Peet, J.T. Pelton, H.A. Schreuder, S. Sunder, C. Tardif, Journal of Medicinal Chemistry 41 (1998) 2461-2480.

[19] T.F. Oliveira, J. Mulchande, R. Moreira, J. Iley, M. Archer, Protein and Peptide Letters 14 (2007) 93-95.

[20] S. Cerqueira Barros, J.A. Martins, J.C. Marcos, A. Cavaco-Paulo, Enzyme Microbial Technology (2012) 107-114.

[21] S. Cerqueira Barros, J.A. Martins, J.C. Marcos, A. Cavaco-Paulo, Peptide Science 98 (2012) 576-590.

[22] A.B. Brauer, G. Kelly, S.J. Matthews, R.J. Leatherbarrow, Journal Biomolecular Structure Dynamic 20 (2002) 59-70.

[23] A.B.E. Brauer, G. Kelly, J.D. McBride, R.M. Cooke, S.J. Matthews, R.J. Leatherbarrow, Journal of Molecular Biology 306 (2001) 799-807.

[24] D.M. Jeffrey, N.M.F. Hamzah, J.L. Robin, European Journal of Biochemistry 266 (1999) 403-412.

[25] J.M. Lizcano, M. Deak, N. Morrice, A. Kieloch, C.J. Hastie, L. Dong, M. Schutkowski, U. Reimer, D.R. Alessi, Journal of Biological Chemistry 277 (2002) 27839-27849

[26] D. Marion, K. Wüthrich, Biochemical and Biophysical Research Communications 113 (1983) 967-974.

[27] A. Bax, D.G. Davis, Journal of Magnetic Resonance 65 (1985) (1969) 355-360.

[28] A. Kumar, R.R. Ernst, K. Wüthrich, Biochemical and Biophysical Research Communications 95 (1980) 1-6.

[29] A.G. Palmer, D.J. Patel, Structure 10 (2002) 1603-1604.

[30] T.D. Goddard, D.G. Kneller, SPARKY 3, University of California, San Francisco, 2006.

[31] K. Wüthrich, NMR of Proteins and Nucleic Acids, Wiley-Interscience, New York, 1986

[32] K. Wüthrich, G. Wider, G. Wagner, W. Braun, Journal of Molecular Biology 155 (1982) 311-319.

[33] P. Güntert, C. Mumenthaler, K. Wüthrich, Journal of Molecular Biology 273 (1997) 283-298.

[34] R.A. Laskowski, M.W. MacArthur, D.S. Moss, J.M. Thornton, Journal of Applied Crystallography 26 (1993) 283-291.

[35] R.A. Laskowski, J.A.C. Rullmann, M.W. MacArthur, R. Kaptein, J.M. Thornton, Journal of Biomolecular NMR 8 (1996) 477-486.

[36] R. Koradi, M. Billeter, K. Wüthrich, Journal of Molecular Graphics 14 (1996) 51-55.

[37] W.L. DeLano, The PyMOL Molecular Graphics System, version 1.2b5, 2004

[38] O. Trott, A.J. Olson, Journal of Computational Chemistry 31 (2010) 455-461.

[39] R. Linding, L.J. Jensen, F. Diella, P. Bork, T.J. Gibson, R.B. Russell, Structure (London, England : 1993) 11 (2003) (1993) 1453-1459.

[40] T. Imamura, N. Yamamoto, A. Tamura, S. Murabayashi, S. Hashimoto, H. Shimada, S. Taguchi, Biochemical and Biophysical Research Communications 369 (2008) 609-615.

[41] B. Legrand, M. Laurencin, J. Sarkis, E. Duval, L. Mouret, J.-F. Hubert, M. Collen, V. Vié, C. Zatylny-Gaudin, J. Henry, M. Baudy-Floc'h, A. Bondon, Biochimica et Biophysica Acta (BBA) - Biomembranes, 1808 (2011) 106-116.

[42] F. Kametani, T. Nonaka, T. Suzuki, T. Arai, N. Dohmae, H. Akiyama, M. Hasegawa, Biochemical and Biophysical Research Communications 382 (2009) 405-409.

[43] W.-H. Cho, Y.-J. Lee, S.-I. Kong, J. Hurwitz, J.-K. Lee, Proceedings of the National Academy of Sciences 103 (2006) 11521-11526.

[44] K. Jaquet, R. Thieleczek, L.M.G. Heilmeyer, European Journal of Biochemistry 231 (1995) 486-490.

[45] M. Edelson-Averbukh, R. Pipkorn, W.D. Lehmann, Analytical Chemistry 79 (2007) 3476-3486.

[46] M.J. Hubbard, P. Cohen, Trends in Biochemical Sciences 18 (1993) 172-177.

[47] S. Megy, G. Bertho, J. Gharbi-Benarous, F. Baleux, R. Benarous, J.-P. Girault, Peptides 26 (2005) 227-241.

[48] N. Evrard-Todeschi, J. Pons, J. Gharbi-Benarous, G. Bertho, R. Benarous, J.-P. Girault, Journal of Chemical Information and Modeling 48 (2008) 2350-2361.

[49] A. Vasconcelos, S. Barros, A. Cavaco-Paulo, Biotechnology (2009) 273-274.

[50] A. Vasconcelos, A.P. Pêgo, L. Henriques, M. Lamghari, A. Cavaco-Paulo, Biomacromolecules 11 (2010) 2213-2220.

[51] A. Vasconcelos, G. Freddi, A. Cavaco-Paulo, Biomacromolecules 9 (2008) 12991305. 